\title{
Probing Molecular Dynamics by Laser-Induced Backscattering Holography
}

\author{
Marko Haertelt, ${ }^{1, *}$ Xue-Bin Bian, ${ }^{2}$ Michael Spanner, ${ }^{1}$ André Staudte, ${ }^{1}$ and Paul B. Corkum ${ }^{1, \uparrow}$ \\ ${ }^{1}$ Joint Attosecond Science Laboratory, University of Ottawa and National Research Council, Ottawa, Ontario, Canada K1A OR6 \\ ${ }^{2}$ State Key Laboratory of Magnetic Resonance and Atomic and Molecular Physics, Wuhan Institute of Physics \\ and Mathematics, Chinese Academy of Sciences, WuHan, HuBei 430071, People's Republic of China
}

(Received 15 September 2015; published 1 April 2016)

\begin{abstract}
We use differential holography to overcome the forward scattering problem in strong-field photoelectron holography. Our differential holograms of $\mathrm{H}_{2}$ and $\mathrm{D}_{2}$ molecules exhibit a fishbonelike structure, which arises from the backscattered part of the recolliding photoelectron wave packet. We demonstrate that the backscattering hologram can resolve the different nuclear dynamics between $\mathrm{H}_{2}$ and $\mathrm{D}_{2}$ with subangstrom spatial and subcycle temporal resolution. In addition, we show that attosecond electron dynamics can be resolved. These results open a new avenue for ultrafast studies of molecular dynamics in small molecules.
\end{abstract}

DOI: 10.1103/PhysRevLett.116.133001

Watching nuclear and electron dynamics in molecules as they occur is a major challenge for science. Significant efforts are underway to follow molecular dynamics using time-resolved x-ray scattering or diffraction [1,2], ultrafast and laser-induced electron diffraction [3-7], and high harmonic generation [8-10]. Strong-field photoelectron holography ( $\mathrm{SFH})$ has been suggested as another avenue to time-resolved imaging [11-13].

SFH where reference and signal waves are born in the same quarter cycle of the laser field has provided insight into the continuum electron wave packet and the ionization process [12-14], but not molecular structure. For diatomics and small molecules, the long-range Coulomb potential contributes most to the total scattering cross section $[15,16]$, and approaches in the forward direction the one-center Rutherford scattering [16]. Short-ranged effects that reflect the molecular structure are overshadowed by this orders-of-magnitude more intense channel. Forward scattering is the origin of the spider structure in all SFH measurements to date.

Signal and reference electron waves can also be born in different quarter cycles, leading to different holographic structures, encoding subcycle dynamics [17]. Specifically, the holographic interference of an unscattered wave with a backscattered electron wave has been proposed as a particularly sensitive probe of the molecular structure [18]. To extract this backscattering interference, the forward scattering problem needs to be overcome. A solution can be found in the photoelectron holography of surfaces. There, the forward scattering peak can be removed in a difference hologram by exploiting its relative weak dependence on the photon energy $[19,20]$. Recent calculations on electron- $\mathrm{H}_{2}^{+}$ scattering [15] suggest a similar approach for SFH. For large scattering angles the differential cross section deviates from the pure Coulomb scattering and furthermore shows an alignment dependence [15]. By calculating the difference between the normalized photoelectron holograms for aligned and antialigned molecules, alignment independent interferences are removed. For the case of hydrogen, the ionization occurs to the electronic ground state only [21]. Therefore, the difference hologram carries primarily information about the difference in backscattering cross sections.

We report differential holograms of $\mathrm{H}_{2}$ and $\mathrm{D}_{2}$. A fishbonelike structure in these holograms is identified to originate from backward scattering. The light mass of $\mathrm{H}_{2}$ makes it a model candidate to study ultrafast nuclear dynamics on the sub-laser-cycle time scale. We observe differences in the differential holograms between hydrogen and deuterium. However, we find that the electronic state of the ion has the largest influence on the hologram. Thus, backscattering holography presents a path to imaging subcycle electron dynamics.

The basic principle of the experiment is shown in Fig. 1. Strong field ionization of $\mathrm{H}_{2}$ in a linearly polarized laser beam creates a continuum electron wave packet, which produces the hologram on a subcycle time scale [Fig. 1(a)]. Simultaneously, a nuclear wave packet in the $1 s \sigma_{g}$ state of the cation is created. In the presence of the laser field, for parallel aligned molecules the $1 s \sigma_{g}$ and $2 p \sigma_{u}$ states couple, leading to dissociation into $\mathrm{H}^{+}$and a neutral hydrogen atom [Fig. 1(b)]. Depending on the order of the coupling, a lower [bond softening (BS)] and a higher momentum [above-threshold dissociation (ATD)] channel [21] are observed [Fig. 1(c)]. As indicated in the figure, the different channels correlate with different parts of the wave packet. We use a newly built cold target recoil ion momentum spectrometer apparatus [22,23] to measure the threedimensional (3D) photoelectron and photoion momenta of all fragments in coincidence. This allows us to distinguish the BS and ATD channel from other ionization or dissociation channels in hydrogen, and, furthermore, we will use it to establish postalignment.

A Ti:sapphire femtosecond laser system $(800 \mathrm{~nm}, 30 \mathrm{fs}$, $2 \times 10^{14} \mathrm{~W} / \mathrm{cm}^{2}, 10 \mathrm{kHz}$ repetition rate) is used for 
(a)

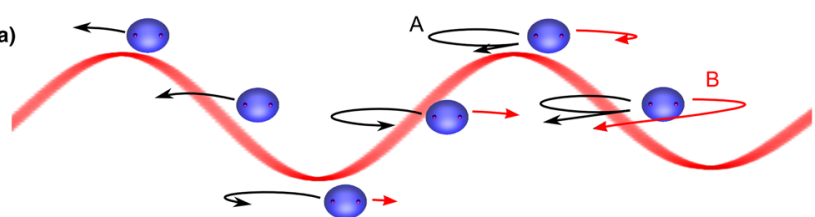

(b)
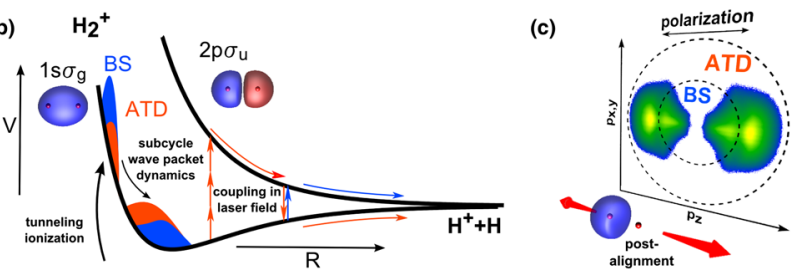

FIG. 1. (a), Sketch of the experiment: $\mathrm{H}_{2}$ is tunnel ionized in a strong laser field. Depending on the time of ionization, different electron trajectories are possible. In the oscillating laser field it may come to backscattering at return (case A). These electrons interfere with indirect electrons started half a laser cycle later (case B). Simultaneously, a wave packet is started in the $1 s \sigma_{g}$ state of $\mathrm{H}_{2}^{+}$(b). In the presence of the field, this state couples to the dissociative $2 p \sigma_{u}$ state, which may lead to dissociation. Depending on the observed proton momentum these are associated to BS and ATD (c). The direction of the proton in the laboratory frame is used for postalignment.

ionization. The linearly polarized pulse was focused with a parabolic mirror into the interaction region with a thin, cold gas target, a 1:1 mixture of $\mathrm{H}_{2}$ and $\mathrm{D}_{2}$.

Count rates have been kept low, $\approx 0.12$ ion hits/shot, to avoid the presence of multiple molecules in the interaction region. The 3D electron distributions are measured for hydrogen and deuterium classifying each event as BS or
ATD by the magnitude of the correlated proton momentum [33]. The recorded 3D proton momentum also defines the molecular alignment in space, i.e., the polar angle $\theta$ between the molecular axis of the neutral molecule at the instant of ionization and the polarization direction $z$ [34].

We consider two molecular alignments, aligned $\left(\theta \leq 10^{\circ}\right)$ and antialigned $\left(\theta \geq 25^{\circ}\right)$ [23]. Because of the selectivity of the dissociation step no protons are observed in the perpendicular direction as can also be seen in Fig. 1(c). The alignment distributions can be characterized by the expectation value of the alignment $\operatorname{cosine}\left\langle\cos ^{2}(\theta)\right\rangle$. In the aligned case, we obtain an expectation value of $0.9936 \pm 0.0003$, and in the antialigned case we obtain $0.787 \pm 0.008$ [35]. Whereas the parallel alignment is very good, a better antialignment would increase the contrast of the measurement. By measuring hydrogen and deuterium simultaneously, we ensured that intensity changes, beam pointing fluctuations, etc., affect both targets and have no influence on the outcome of the experiment.

In Fig. 2(a) we show the measured electron momentum distribution (we use atomic units throughout the Letter) for aligned $\mathrm{D}_{2}$ from ATD (for the other channels see Ref. [23]). This represents the strong field hologram. The basic features that can be identified are above-threshold ionization (ATI) rings (feature A) and the spider structure (feature B) originating in forward scattering holography $[12,14]$. In the initial correlated photoelectron momentum distributions, we made use of the cylindrical symmetry along the polarization direction. However, we did not use inversion symmetry in the $z$ direction.
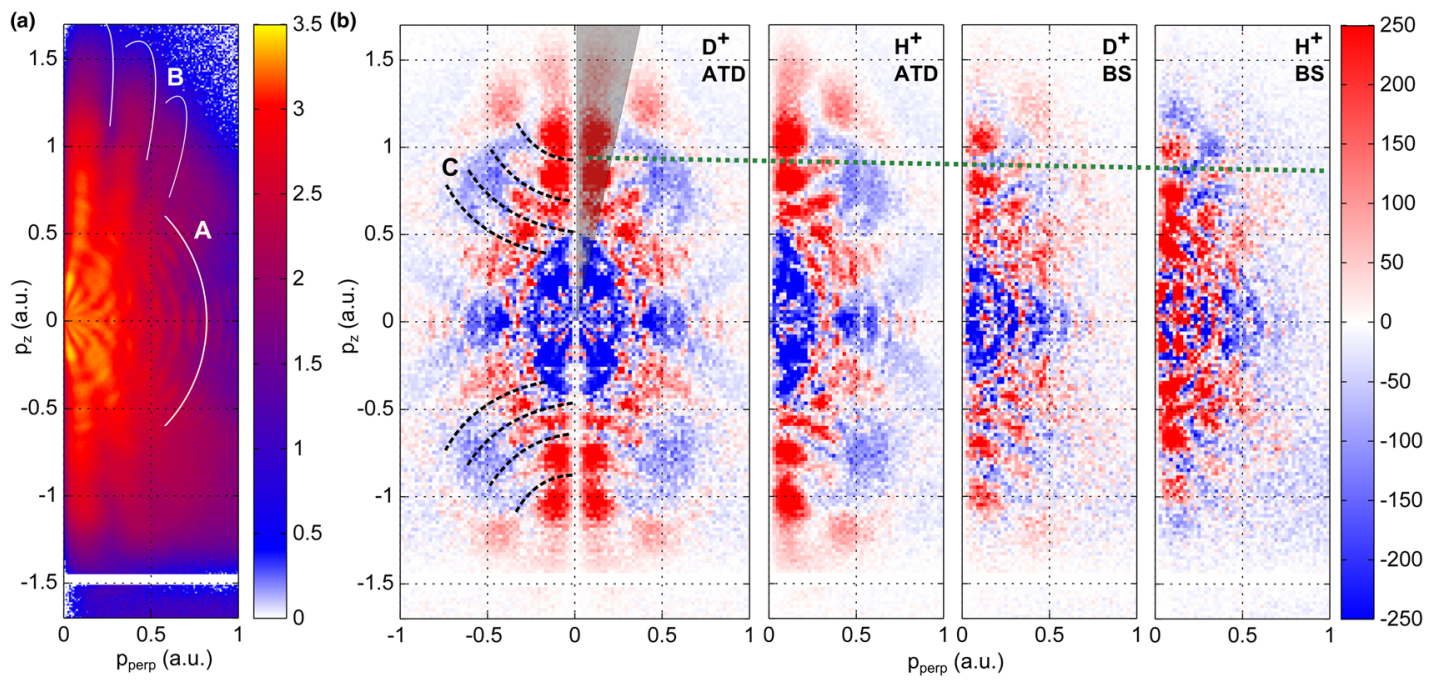

FIG. 2. (a), Measured 3D electron momentum distribution for aligned $\mathrm{D}_{2}$ molecules leading to ATD. The intensity is plotted on a logarithmic scale. The ATI and the spider structure from forward scattering photoelectron holography are marked as features A and B, respectively. (b) Differential holograms of the measured photoelectron momentum distributions between the aligned and antialigned molecules correlated with BS or ATD in $\mathrm{H}_{2}$ or $\mathrm{D}_{2}$. The normalized difference for the ATD channel of deuterium was mirrored. The fishbone structure is indicated as feature $\mathrm{C}$. The shaded area shows the part of the photoelectron distribution used for the projection in Fig. 3(d). The fringe shift is highlighted by the green dotted line. 
For each case, i.e., BS and ATD in hydrogen and deuterium, we calculated the difference between the normalized photoelectron distributions of the aligned and antialigned molecules in Fig. 2(b) (for details and a binwise normalized difference see Ref. [23]). This is our differential hologram. For illustration purposes we mirrored the differential hologram for the ATD channel of $\mathrm{D}_{2}$. A fishbonelike structure (feature $\mathrm{C}$ ) is revealed. This structure is only weakly visible in the original hologram. The same feature is also observed in the other channels. We have confirmed the equal intensity using the ATI rings [23] and corrected for the small differences in the proton alignment distributions between the different considered channels; however, no significant changes in the normalized differences are observed.

Previous theoretical work based on the strong-field approximation identified two mechanisms that may lead to such a fishbone structure [17]: the temporal double slit [36-40] and backscattering holography [17]. The former is caused by the interference of a direct and an indirect electron wave packet ionized within a half cycle of the laser pulse. Particularly, the temporal double slit assumes that the parent ions' Coulomb potential can be neglected. In the case of hydrogen, for which the ionization step is almost isotropic [41], no alignment dependence is expected for the double-slit interference. In contrast, backscattering holography naturally provides an alignment dependence in the recollision step with the parent ion. In the following, we discuss our results with respect to the latter mechanism.

In Figs. 3(a)-3(c) we show the results of a semiclassical model $[17,18,23,42]$, calculating the phase difference between the backscattered and reference electron wave. We only consider the backscattering holography channel that is predicted to be dominant [43]. Specifically, the signal wave is created by an electron trajectory ionized near the peak of the electric field (a long trajectory, using the nomenclature from high harmonics generation). The reference wave is an indirect electron trajectory that is ionized in the third quarter cycle and is turned around in the laser field.

In our semiclassical model backscattering occurs at the individual nuclei of the molecule. Thus, a phase shift is introduced into the hologram depending on the molecular site where the electron backscatters. In Figs. 3(a) and 3(b) we show the hologram for backscattering on the parent or neighboring atom, respectively, placed at a distance of 2 a.u. for $\mathrm{H}_{2}^{+}$aligned parallel to the laser polarization. The phase difference plots qualitatively agree with our experimentally observed fishbone structure. In the Supplemental Material [23], we show measurements for the ATD channel of $\mathrm{H}_{2}$ for two more intensities and the corresponding predictions of the semiclassical model.

The phase shift introduced due to scattering on the neighboring site depends on the internuclear separation, as can be seen in Fig. 3(c), which shows the phase difference
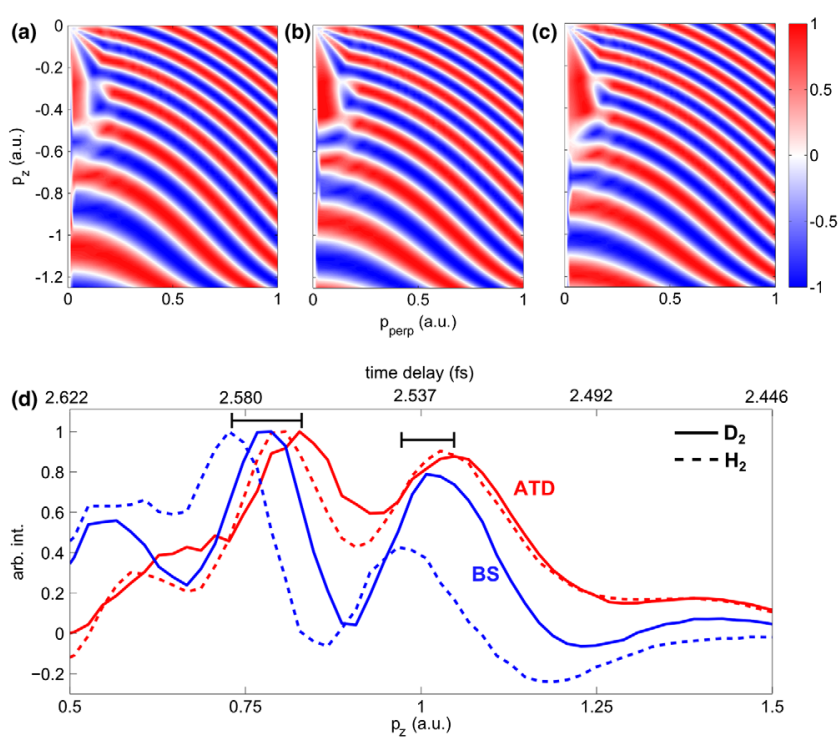

FIG. 3. (a)-(c) Phase difference between the signal and reference electron wave as predicted by a semiclassical model for backscattering holography [18]. (a) Backscattering on parent ion at $R_{0}$ equal to 2.0 a.u. (b),(c) Backscattering on neighboring ion at $R_{0}$ equal to 2.0 a.u. (b) and 3.5 a.u. (c). (d) Projection of experimental data as indicated by the gray area in Fig. 2(b). The black, horizontal bars indicate the range of the two strongest interference fringes.

for an internuclear separation of 3.5 a.u. The comparison of Figs. 3(b) and 3(c) reveals that a larger internuclear separation results in more fringes and a fringe shift to smaller final momenta.

The above suggests that the position of the interference fringes gives information about the nuclear subcycle dynamics. Between ionization and recollision the molecule starts dissociating. For a long trajectory electron, which probes the molecule by backscattering, ionization occurs close to the peak of the electric field of the laser pulse. Recollision takes place almost one optical cycle later. The nuclear dynamics for hydrogen is faster than for deuterium under otherwise almost identical ionization energies and bond distances for their neutral molecules. The differential holograms in Fig. 2(b) exhibit the isotopic fringe shift in each of the different dissociation channels. Additionally, we observe a shift of the fringes between ATD and BS. This shift indicates that the internuclear separation $R$ at the time of recollision is $R_{\mathrm{BS}}^{H}>R_{\mathrm{BS}}^{D}>R_{\mathrm{ATD}}^{H}>R_{\mathrm{ATD}}^{D}$.

Since the fringe shift is strongest along the polarization direction, we project all perpendicular momenta with a polar angle smaller than $10^{\circ}$ with respect to the $z$ direction onto $p_{z}$ [shaded region in Fig. 2(b)] and show them in Fig. 3(d). We again observe only a small isotopical shift, but a large shift between the two dissociation pathways. Furthermore, the fringes experience a stronger shift at a smaller momentum. 

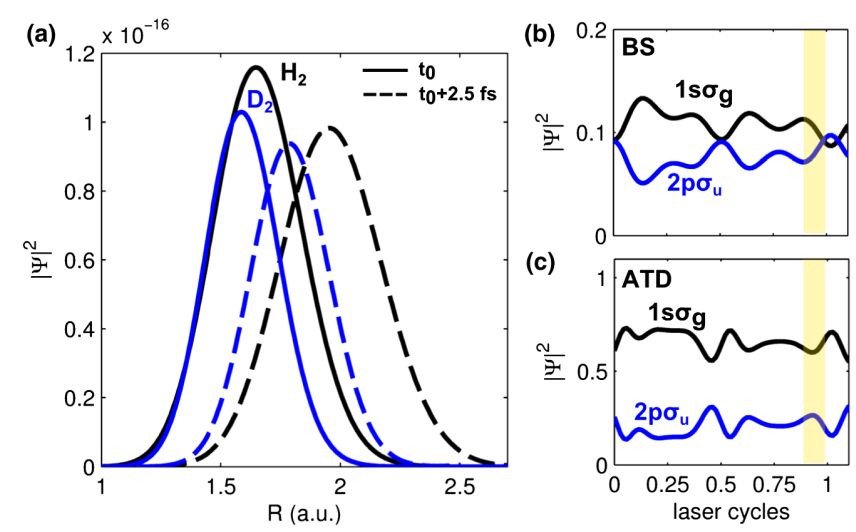

FIG. 4. Nuclear wave packet simulation for a 1D two-surface model of hydrogen. (a) Nuclear wave packet for hydrogen and deuterium in black and blue, respectively, at the time of ionization $t_{0}$ and for a recollision time of $2.5 \mathrm{fs}$. In (b) and (c), the population in the $2 p \sigma_{u}$ and $1 s \sigma_{g}$ state leading to BS and ATD, respectively. The final population in the $1 s \sigma_{g}$ state was used for normalization and is corrected by the bound state contributions. The highlighted area shows the recollision time of the signal electron.

We use the time-dependent Schrödinger equation for the nuclear wave packet propagation in hydrogen and deuterium [23] and the semiclassical model to estimate the expected fringe shift seen with backscattering holography. For an on-axis $p_{z}=1.0$ a.u. signal electron the time delay between ionization and recollision is $\approx 2.5$ fs. During that time, the different nuclear wave packet dynamics causes a difference in bond length of $\Delta R=0.2$ a.u. [Fig. 4(a)]. Such a bond length difference transforms into a fringe shift of 0.013 a.u. for the interference fringe at $p_{z}=1$ a.u. This agrees well with the experimental shift for the ATD channel, which is $0.008 \pm 0.004$ a.u. However, the larger shift for the BS channel of $0.049 \pm 0.004$ a.u. cannot be explained with a change in internuclear distance alone (for fitted peak positions see Ref. [23]).

Now we turn to the momentum dependence of the holographic fringes. The maximum in Fig. 3(d) at $p_{z}=$ $\sim 0.8$ a.u. shows a larger dependence on the dissociation channel and the isotope than the maximum at $p_{z}=$ $\sim 1.0$ a.u. In contrast, the semiclassical model predicts the shift to be proportional to the momentum [18]. For a long trajectory electron, the time delay between ionization and recollision is larger for smaller final electron momenta. However, the model does not include any electron or nuclear dynamics that could occur within that time frame. The difference in time delay between ionization and recollision for the 0.8 and 1.0 a.u. final electron momentum is $\sim 50$ as. Our nuclear wave packet simulation confirms that there is no significant change in bond distance for such a short time window.

Therefore, we turn to electron dynamics, which determines the dissociation channel. To understand the subcycle electron dynamics directly after ionization we used a "postselection" strategy. Using our nuclear wave packet simulation, we have first assigned the different parts of the wave packet to their final state, i.e., the bound contribution, $\mathrm{BS}$, or ATD. We propagated the wave packet to a time after the full laser pulse, and split the final wave packet into its different components $|\psi\rangle=\left|\psi_{\mathrm{BS}}\right\rangle+\left|\psi_{\mathrm{ATD}}\right\rangle+\left|\psi_{\text {bound }}\right\rangle$. Second, each individual wave packet was propagated backwards in time.

In Figs. 4(b) and 4(c) we show the populations in the $\sigma_{u}$ and $\sigma_{g}$ states as a function of the laser cycle after ionization leading to BS and ATD, respectively. Whereas ATD is dominated by the ground state, both states contribute to BS similarly. The $\sigma_{g}$ and $\sigma_{u}$ states not only have different total backscattering cross sections but also have a different angular dependence [15]. A closer look at the normalized differences in Fig. 2(b) reveals not only shifts of the backscattering holography fringes, but also different angular distributions.

Furthermore, we show in Figs. 4(b) and 4(c) that for both dissociation channels a coupling of the two electronic states begins directly after ionization. It is strongest at the maxima of the field and causes a deformation of the orbital due to polarization. The highlighted area shows the recollision time of the signal-wave photoelectron. Signal electrons with smaller final momenta are backscattered closer to the field maximum and are therefore more affected by polarization. This explains the fringe shift dependence on the observed photoelectron momentum.

Concluding, differential holography is a powerful method. Elusive details of tunnel ionization have previously been revealed by comparing holograms from different molecular alignment distributions [13]. Yet, the dominant forward scattering has prevented any molecular structure to be resolved. Here, we have shown that by conserving the cylindrical symmetry in the alignment distributions a backscattering hologram will emerge.

The backscattering of low-energy photoelectrons is determined by the spatial distribution of the ion's charges. Therefore, backscattering holograms are sensitive to both the nuclear and electronic charge distribution. While the fast nuclear dynamics between ionization and recollision of the signal wave contributes to the differential hologram, the character of the remaining bound-state wave function has the strongest influence on the differential hologram. For a single electron system, such as $\mathrm{H}_{2}^{+}$, changes of the electronic state result in a large change in the scattering cross sections. This allowed us to differentiate the nascent electronic behavior that leads to the various dissociative ionization channels of strongly driven $\mathrm{H}_{2}$.

Backscattering holography has the potential to not only reveal attosecond electron dynamics, but also dynamic electron-nuclear correlation. Our measurement shows that the subcycle electron dynamics already begins with tunnel ionization of the neutral molecule, and that the initial distribution of electronic states predetermines the dissociation channel. 
There are multiple opportunities to extend the method. First, we rely on the time to energy mapping. This mapping can be controlled by changing the intensity or wavelength of the laser field. Second, our experiment relies on postselected alignment. This is only possible for a few molecular systems. However, active alignment techniques overcome this limitation. Finally, coincidence imaging limits the repetition rate, and therefore the dynamic range, of our measurement. Velocity map imaging and megahertz femtosecond laser sources will allow orders-of-magnitude signal-to-noise improvement, opening differential holography to more complex systems.

M. H. would like to thank the Alexander von Humboldt Foundation for the support of a Feodor-Lynen Fellowship. We would like to thank Andrei Naumov for support with the laser system, Bert Avery and Dave Crane for technical support, and D. M. Villeneuve and P. Hockett for helpful discussions. Financial support from a NSERC discovery grant (RPGIN/419092-2013) is gratefully acknowledged.

*marko.haertelt@uottawa.ca †pcorkum@uottawa.ca

[1] J. Küpper et al., Phys. Rev. Lett. 112, 083002 (2014).

[2] M. P. Minitti, J. M. Budarz, A. Kirrander, J. S. Robinson, D. Ratner, T. J. Lane, D. Zhu, J. M. Glownia, M. Kozina, H. T. Lemke, M. Sikorski, Y. Feng, S. Nelson, K. Saita, B. Stankus, T. Northey, and J. Hastings, Phys. Rev. Lett. 114, 255501 (2015).

[3] R. Srinivasan, J. S. Feenstra, S. T. Park, S. Xu, and A. H. Zewail, Science 307, 558 (2005).

[4] M. Meckel, D. Comtois, D. Zeidler, A. Staudte, D. Pavičić, H. C. Bandulet, H. Pépin, J. C. Kieffer, R. Dörner, D. M. Villeneuve, and P. B. Corkum, Science 320, 1478 (2008).

[5] J. Xu, Z. Chen, A.-T. Le, and C. D. Lin, Phys. Rev. A 82, 033403 (2010).

[6] G. Sciaini and R. J. D. Miller, Rep. Prog. Phys. 74, 096101 (2011).

[7] C. I. Blaga, J. Xu, A. D. DiChiara, E. Sistrunk, K. Zhang, P. Agostini, T. A. Miller, L. F. DiMauro, and C. D. Lin, Nature (London) 483, 194 (2012).

[8] S. Baker, J. Robinson, C. Haworth, H. Teng, R. Smith, C. Chirilă, M. L. L. Tisch, and J. Marangos, Science 312, 424 (2006).

[9] O. Smirnova, Y. Mairesse, S. Patchkovskii, N. Dudovich, D. Villeneuve, P. Corkum, and M. Y. Ivanov, Nature (London) 460, 972 (2009).

[10] H. J. Wörner, J. B. Bertrand, D. V. Kartashov, P. B. Corkum, and D. M. Villeneuve, Nature (London) 466, 604 (2010).

[11] M. Spanner, O. Smirnova, P. B. Corkum, and M. Y. Ivanov, J. Phys. B 37, L243 (2004).

[12] Y. Huismans et al., Science 331, 61 (2011).

[13] M. Meckel, A. Staudte, S. Patchkovskii, D. M. Villeneuve, P. B. Corkum, R. Dörner, and M. Spanner, Nat. Phys. 10, 594 (2014).

[14] D. D. Hickstein, P. Ranitovic, S. Witte, X. M. Tong, Y. Huismans, P. Arpin, X. Zhou, K. E. Keister, C. W. Hogle, B.
Zhang, C. Ding, P. Johnsson, N. Toshima, M. J. J. Vrakking, M. M. Murnane, and K. C. Kapteyn, Phys. Rev. Lett. 109, 073004 (2012).

[15] A. G. Harvey and J. Tennyson, in Quantum Dynamic Imaging, CRM Series in Mathematical Physics, edited by A. D. Bandrauk and M. Ivanov (Springer, New York, 2011), pp. $55-70$.

[16] H. Miyagi, T. Morishita, and S. Watanabe, Phys. Rev. A 85, 022708 (2012).

[17] X.-B. Bian, Y. Huismans, O. Smirnova, K.-J. Yuan, M. J. J. Vrakking, and A. D. Bandrauk, Phys. Rev. A 84, 043420 (2011).

[18] X.-B. Bian and A. D. Bandrauk, Phys. Rev. Lett. 108, 263003 (2012).

[19] S. Omori, Y. Nihei, E. Rotenberg, J. Denlinger, S. Kevan, B. Tonner, M. V. Hove, and C. Fadley, J. Electron Spectrosc. Relat. Phenom. 114-116, 455 (2001).

[20] S. Omori, Y. Nihei, E. Rotenberg, J. D. Denlinger, S. Marchesini, S. D. Kevan, B. P. Tonner, M. A. Van Hove, and C. S. Fadley, Phys. Rev. Lett. 88, 055504 (2002).

[21] J. H. Posthumus, Rep. Prog. Phys. 67, 623 (2004).

[22] J. Ullrich, R. Moshammer, A. Dorn, R. Dörner, L. P. H. Schmidt, and H. Schmidt-Böcking, Rep. Prog. Phys. 66, 1463 (2003).

[23] See Supplementary Material at http://link.aps.org/ supplemental/10.1103/PhysRevLett.116.133001 for experimental details, data for other intensities, ATI peak analysis, and details about the theoretical modeling, which includes Refs. [24-32].

[24] P. B. Corkum, Phys. Rev. Lett. 71, 1994 (1993).

[25] A. D. Bandrauk, S. Chelkowski, and S. Goudreau, J. Mod. Opt. 52, 411 (2005).

[26] M. Busuladžić, A. Gazibegović-Busuladžić, D. B. Milošević, and W. Becker, Phys. Rev. Lett. 100, 203003 (2008).

[27] N. Takemoto and A. Becker, Phys. Rev. Lett. 105, 203004 (2010).

[28] G. G. Paulus, W. Becker, W. Nicklich, and H. Walther, J. Phys. B 27, L703 (1994).

[29] M. Lein, J. P. Marangos, and P. L. Knight, Phys. Rev. A 66, 051404 (2002).

[30] M. Lewenstein, P. Balcou, M. Y. Ivanov, A. L'Huillier, and P. B. Corkum, Phys. Rev. A 49, 2117 (1994).

[31] A. E. Boguslavskiy, J. Mikosch, A. Gijsbertsen, M. Spanner, S. Patchkovskii, N. Gador, M. J. J. Vrakking, and A. Stolow, Science 335, 1336 (2012).

[32] F. V. Bunkin and I. I. Tugov, Phys. Rev. A 8, 601 (1973).

[33] Proton momenta smaller than 7.2 a. u. for hydrogen and 11.3 a. u. for deuterium are considered to belong to BS and the larger one to ATD (with maximum momenta of 14 and 20 a. u. for hydrogen and deuterium, respectively).

[34] A. Staudte, S. Patchkovskii, D. Pavičić, H. Akagi, O. Smirnova, D. Zeidler, M. Meckel, D. M. Villeneuve, R. Dörner, M. Y. Ivanov, and P. B. Corkum, Phys. Rev. Lett. 102, 033004 (2009).

[35] For each measured proton, we have calculated $\cos ^{2}(\theta)$ using the measured polar angle $\theta$ with respect to the polarization direction. The expectation value given is the mean of the distribution. The error margin indicates the maximum difference between $\mathrm{H}_{2}$ and $\mathrm{D}_{2}$, and is separated for ATD 
and BS. The small error indicates that although the dissociation occurs on a much slower time scale for $\mathrm{D}_{2}$, the alignment distribution is almost unaffected, and therefore should not contribute to any differences observed in the differential holograms.

[36] F. Lindner, M. G. Schätzel, H. Walther, A. Baltuška, E. Goulielmakis, F. Krausz, D. B. Milošević, D. Bauer, W. Becker, and G. G. Paulus, Phys. Rev. Lett. 95, 040401 (2005).

[37] D. G. Arbó, E. Persson, and J. Burgdörfer, Phys. Rev. A 74, 063407 (2006).

[38] R. Gopal, K. Simeonidis, R. Moshammer, T. Ergler, M. Durr, M. Kurka, K. U. Kühnel, S. Tschuch, C. D. Schröter, D. Bauer, J. Ullrich, A. Rudenko, O. Herrwerth, T. Uphues,
M. Schultze, E. Goulielmakis, M. Uiberacker, M. Lezius, and M. F. Kling, Phys. Rev. Lett. 103, 053001 (2009).

[39] X. Xie, S. Roither, D. Kartashov, E. Persson, D. G. Arbó, L. Zhang, S. Gräfe, M. S. Schöffler, J. Burgdörfer, A. Baltuška, and M. Kitzler, Phys. Rev. Lett. 108, 193004 (2012).

[40] M. Richter, M. Kunitski, M. Schöffler, T. Jahnke, L. P. H. Schmidt, M. Li, Y. Liu, and R. Dörner, Phys. Rev. Lett. 114, 143001 (2015).

[41] I. Petersen, J. Henkel, and M. Lein, Phys. Rev. Lett. 114, 103004 (2015).

[42] X.-B. Bian and A. D. Bandrauk, Phys. Rev. A 89, 033423 (2014).

[43] M. Li, J. Yuan, X. Sun, J. Yu, Q. Gong, and Y. Liu, Phys. Rev. A 89, 033425 (2014). 